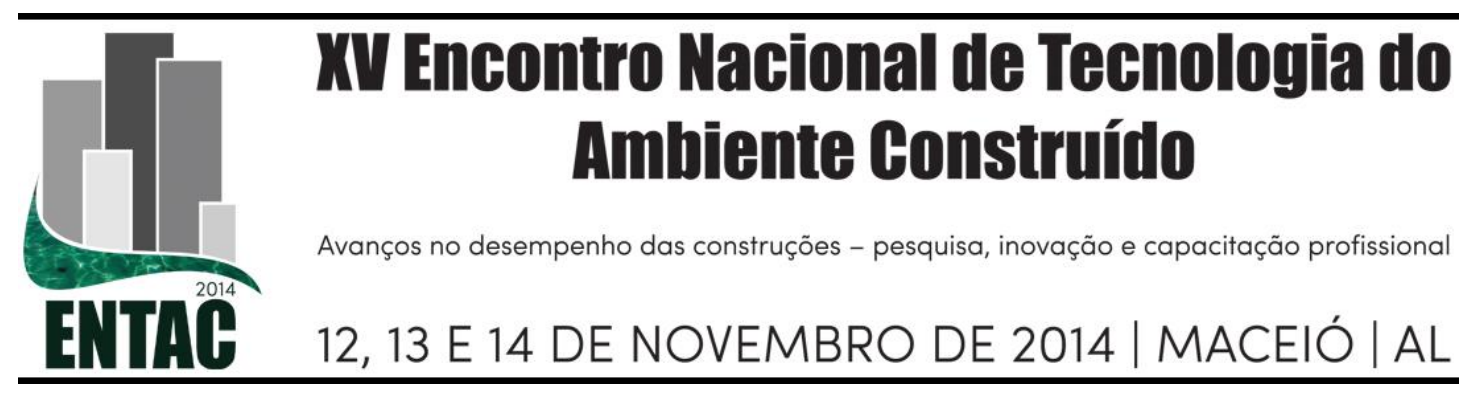

\title{
COMPARAÇÃO DE PROCESSOS DE LEVANTAMENTO DE QUANTITATIVOS: TRADICIONAL E BIM
}

\author{
COSTA, José Martins C. da (1); SERRA, Sheyla M. B. (2)
}

Programa de Pós-Graduação em Estruturas e Construção Civil (PPGECIV), Universidade Federal de São

Carlos (UFSCar), Brasil (1) jmartinscc@ hotmail.com; (2) sheylabs@ufscar.br

\begin{abstract}
RESUMO
O processo de orçamentação na construção civil é fundamental para o sucesso do empreendimento. Como procedimento usual, utiliza-se de planilhas orçamentárias para levantamento de quantitativos, nem sempre compatibilizadas com o projeto e com o processo na construção. Tendo como base o cenário atual do setor da construção civil e adoção de novas tecnologias para a representação de projetos, o Building Information Modeling (BIM) vem assumindo um papel imprescindível na obtenção de produtos com maior nível de confiabilidade projetual. Este artigo tem como propósito comparar o processo de levantamento de quantitativos com base no método tradicional com a extração de informações de um modelo BIM de obras civis. O método de pesquisa usado para o desenvolvimento do artigo foi o estudo de caso da construção do Laboratório de Sustentabilidade e Segurança em Canteiros de Obras do Departamento de Engenharia Civil da UFSCar. Os projetos arquitetônico e estrutural foram concebidos em softwares 2D. Para fins de comparação, os projetos desta obra foram modelados para extração dos quantitativos a partir do BIM e posteriormente foram comparados com os quantitativos calculados manualmente. Foi utilizado para a modelagem o software Sketchup de propriedade da empresa Google. Os resultados indicaram que os quantitativos extraídos do BIM apresentam um alto grau de confiabilidade e que a grande maioria dos itens da planilha orçamentária pode ser extraída de forma automática por meio do modelo BIM. Isto sugere que o BIM é uma ferramenta que não apenas facilita as atividades de projeto, mas também contribui para outras atividades, como orçamento, eliminando os erros manuais comuns do levantamento de quantitativos e colaborando para a qualidade do orçamento.
\end{abstract}

Palavras-chave: Building Information Modeling (BIM), Levantamento de Quantitativos, Orçamentação.

\begin{abstract}
The budgeting process in construction is critical to the success of the venture. As usual procedure is used for budget spreadsheets for quantitative survey, not always aligned to the design and construction process. Based on the current situation in the construction sector and adoption of new technologies for the representation of designs, the Building Information Modeling (BIM) has had an essential role in obtaining products with the highest level of design reliability. This article aims to compare the process of quantitative survey based on the traditional method with the extraction of information from a BIM model of construction works. The research method used to develop the product was the case study of the construction of the Laboratory for Sustainability and Safety in Construction Site of the Department of Civil Engineering of UFSCar. The architectural and structural projects were designed in $2 D$ software. For purposes of comparison, this work designs were modeled for extracting quantitative from BIM and later were compared with quantitative manually computed. Sketchup software owned by Google was used for modeling. The results indicated that quantitative extracted from BIM exhibit a high degree of reliability and the vast majority of items in the budget worksheet can be extracted automatically via the BIM model. This suggests that BIM is a tool that not only facilitates project activities, but contributes to other activities such as budget, eliminating common manual errors on the quantitative takeoff and helps the estimating quality.
\end{abstract}

Keywords: Building Information Modeling (BIM), Quantitative takeoff, Budgeting. 


\section{INTRODUÇÃO}

No atual cenário empresarial, o valor de produtos e serviços é determinado pelo mercado, se contrapondo ao passado, onde os mesmos eram estabelecidos em função do lucro desejado ou esperado pelas empresas. Isso submete a indústria da construção civil a buscar uma gestão de processos adequada, que tenha como foco a redução e/ou eliminação de atividades que não agregam valor. Deste modo, o lucro passou a depender, muito constantemente, da redução de custo, e com isso, a orçamentação, o planejamento e a gestão de custos tem assumido um importante valor para a sobrevivência das empresas atuantes neste ramo.

As empresas de construção civil vêm sendo obrigadas a evoluir em sua forma de gerenciamento para garantir a sobrevivência no mercado. $\mathrm{O}$ atual cenário econômico induz as empresas a adotarem o uso de novas tecnologias de informação e comunicação e de novos modelos de administração, a fim de obter uma melhor produtividade, rentabilidade e manter um bom nível de qualidade. Dentre as inovações tecnológicas se destaca o Building Information Modelling (BIM).

O BIM vem assumindo um papel importante na obtenção de produtos com maior nível de confiabilidade projetual, pois o mesmo pode contribuir para obtenção de projetos com maior qualidade, com compatibilização de projetos, com melhor intercâmbio de informações, com levantamento mais preciso de quantitativos, com a possibilidade de simulação do planejamento, entre outros aspectos (EASTMAN, TEICHOLZ, SACKS, \& LISTON, 2014). Todos estes fatores contribuem para alimentar a precisão do sistema de informação e, consequentemente, melhorar o processo de tomada de decisão.

Utilizado inicialmente na fase de análise de viabilidade do empreendimento, o orçamento é uma de suas principais ferramentas gerenciais e possui diferentes características ao longo do seu desenvolvimento. Pode ser determinado com base em estimativas de custo (gerais ou por etapa de obra) ou ser detalhado de forma a considerar todos os serviços da obra. Neste caso, deve-se fazer um levantamento detalhado dos quantitativos de materiais, serviços e equipamentos envolvidos.

Para a concepção da base de quantitativos que serve ao orçamento utiliza-se de várias informações, como os projetos executivos, os critérios de medição de áreas, volumes e serviços, os índices de dimensionamento do trabalho e o conhecimento sobre a tecnologia e técnica do serviço construtivo a ser executado. A demanda de serviços apresentada para o orçamentista consiste de várias etapas, como leitura de projeto, definição de critérios de medição, consulta a tabelas de indicadores de engenharia, tabulação de números, coleta de preços, entre outros. Como procedimento usual, são concebidas planilhas orçamentárias, que procuram registrar as medidas observadas durante esta etapa da orçamentação. Porém, apesar da prática intensiva dessa forma de se obter as quantidades de serviços e insumos, verifica-se o surgimento de inúmeros problemas no processo de elaboração de orçamentos na construção civil, dentre eles podem ser destacados: falhas e incompatibilidade de projetos, planejamento inadequado, erros no levantamento de quantitativos, falta de informações sobre os índices de dimensionamento, que podem ocasionar elevação nos custos de obra e um processo de compras inadequado (MATTOS, 2006).

Para Andrade (2012), é visível a inconsistência direta entre os sistemas bidimensionais de representação e a extração dos dados quantitativos em projetos. Para essa autora, a contribuição do BIM na fase de levantamento de quantitativos resulta em três vantagens: maior precisão com a extração automática das tabelas de quantitativos; proporcionar maior necessidade de reflexão sobre a tecnologia da construção para o projetista ou arquiteto; evolução da forma de projetar visando eficiência no processo. 
Para Santos et al. (2009), o levantamento de quantitativos pode ser realizado tanto manualmente quanto eletronicamente, dependendo da preferência e das ferramentas disponíveis pelo orçamentista. Para esses autores, os métodos tradicionais de se realizar um levantamento incluem a medição de todos os elementos de um edifício, utilizandose da escala ou da leitura de medidas em projeto. Além ser um método que pode ser considerado "tedioso", requer muita atenção na transferência das medidas para a planilha de quantitativos, gerando necessidade de um processo de conferência.

Segundo Sabol (2008), a estimativa de custo de projetos de construção começa tradicionalmente com a quantificação - um processo demorado onde se é analisado conjuntos impressos de desenho, ou mais recentemente, projetos em CAD. A partir destas quantidades, os orçamentistas utilizam planilhas para obter o custo estimado do projeto. Para essa autora, o processo de quantificação está sujeito a erro humano, tende a propagar imprecisões, e consume uma considerável parcela de tempo - em torno de $50 \%$ a $80 \%$ do tempo do orçamentista num determinado projeto.

Assim, torna-se importante refletir sobre esta importante etapa do processo de orçamentação. Este artigo tem como propósito analisar a contribuição do BIM para o processo orçamentário de levantamento de quantitativos de obras civis, fazendo uma comparação entre os dados obtidos via processo tradicional de leitura de projeto $2 \mathrm{D}$ com o processo de medição por meio do BIM.

\section{ORÇAMENTO}

Segundo Barbosa et al. (2009), orçamentação é estabelecer, a partir do escopo e cronograma, uma projeção de recursos monetários necessários para execução dos serviços de um projeto, agrupados de forma a explicar, da melhor forma possível, o custo total do projeto e sua apropriação no eixo do tempo. Portanto, o custo total deverá ser subdividido em seus principais componentes, alocados nos pacotes de trabalhos, contas de controle e produtos intermediários, ao logo do cronograma do projeto, possibilitando ter uma referência para acompanhar a evolução do mesmo.

De acordo com Mattos (2006), de forma esquemática o orçamento tem três etapas de trabalho: estudo das condicionantes, composição de custos e determinação do preço, como descrito na Quadro 1.

\section{Quadro 1 - Etapas do processo de orçamentação}

\begin{tabular}{|l|l|}
\hline \multicolumn{1}{|c|}{ ETAPAS } & \multicolumn{1}{c|}{ SUBETAPAS } \\
\hline \multirow{3}{*}{ Estudo das condicionantes } & $\begin{array}{l}\text { - Leitura e interpretação dos projetos e especificações técnicas; } \\
\text { - Leitura e interpretação do edital; } \\
\text { - Visita técnica. }\end{array}$ \\
\hline \multirow{3}{*}{ Composição de Custos } & $\begin{array}{l}\text { - Identificação dos serviços; } \\
\text { - Levantamento de Quantitativos; } \\
\text { - Descrição dos custos diretos; } \\
\text { - Descrição dos custos indiretos; } \\
\text { - Cotação de preços; } \\
\text { - Definição de encargos sociais e trabalhistas. }\end{array}$ \\
\hline \multirow{3}{*}{ Fechamento do orçamento } & $\begin{array}{l}\text { - Definição da lucratividade; } \\
\text { - Cálculo do BDI; } \\
\text { - Desbalanceamento da planilha. }\end{array}$ \\
\hline
\end{tabular}

Fonte: Mattos (2006)

Para Santos et al. (2009), os orçamentos tradicionais geralmente dividem os custos da obra em custos diretos e indiretos. Os custos diretos são aqueles referentes aos insumos 
da obra, como, por exemplo, os custos de materiais, equipamentos, mão-de-obra e encargos sociais, sendo os custos indiretos aqueles referentes à administração, ao financiamento e aos impostos. Segundo esses autores, é importante que o orçamento seja adequado à estratégia de execução do empreendimento e que possa ser periodicamente atualizado.

\section{BUILDING INFORMATION MODELLING (BIM)}

Um empreendimento atualmente tem seu processo de implementação fragmentado em várias disciplinas e a comunicação entre elas é baseada em papel e em 2D. Deste modo, podem ser gerados erros e omissões nos documentos resultando em custos inesperados, atrasos na data de entrega e eventuais litígios judiciais entre as diversas partes envolvidas. Assim, o BIM é um dos esforços recentes para tratar esses problemas (EASTMAN, 2014).

O BIM é conceituado por Eastman (2014) como uma metodologia de modelagem e processos integrados para produzir, analisar e comunicar modelos com as informações de edifícios. E tem como características principais o uso de modelos paramétricos e facilitar a comunicação e colaboração entre os profissionais da construção civil. Através da criação do modelo integrado virtual da construção, é possível integrar todas as disciplinas e compreender todo o ciclo de vida da edificação, como também gerar de forma automática, documentos, desenhos, quantitativos, entre outras informações (EASTMAN, 2014).

O BIM atua em uma base de dados virtual possibilitando que qualquer alteração feita na base reflita em todas as peças desenhadas, possibilitando que os participantes do ciclo de vida do empreendimento possam visualizar o modelo, conseguindo facilmente compartilhar e sincronizar informações (AZEVEDO, 2009).

A modelagem 3D vinculada ao orçamento é denominada como modelagem 5D e é caracterizada por acrescentar o custo ao modelo virtual. Tendo com principal objetivo obter o custo do empreendimento, aumentando a precisão durante a construção, tendo menos desperdício de tempo e de materiais, e redução da quantidade de alterações durante a execução das obras devido a maior conciliação das especialidades (AZEVEDO, 2009).

De acordo com Witicovski (2011), o BIM não se caracteriza pelo uso de novas ferramentas de software, mas por um novo enfoque de negócios e de uma nova estrutura organizacional das empresas, que é configurada de uma forma inovadoro, onde as pessoas trabalham juntas em um tempo real. Segundo a mesma autora, o uso estratégico da tecnologia da informação e do BIM, mediante integração de dados, informação, controle e processo deve ser analisado dentro do sistema gerencial inserido.

Desde modo, com a adoção do BIM pode-se gerar informações mais seguras para o planejamento e o gerenciamento de custo do empreendimento possibilitando aos gestores uma visão e compreensão facilitada dos prazos e custos do empreendimento.

\section{METODOLOGIA}

A metodologia usada para o desenvolvimento da pesquisa foi o estudo de caso da construção do Laboratório de Sustentabilidade e Segurança em Canteiros de Obras do Departamento de Engenharia Civil da Universidade Federal de São Carlos (UFSCar). A escolha deste edifício foi motivada pela obrar possuir arquitetura simples, estrutura convencional de concreto armado e pela mesma estar inserida dentro do Campus São Carlos-SP, como destacado com o símbolo na cor vermelha na Figura 1. A edificação estudada é caracterizada por ser uma obra térrea composta por duas salas com a 
concepção de laboratórios secos, como descritos na planta baixa e no corte ilustrado na Figura 2.

Figura 1 - Localização do laboratório

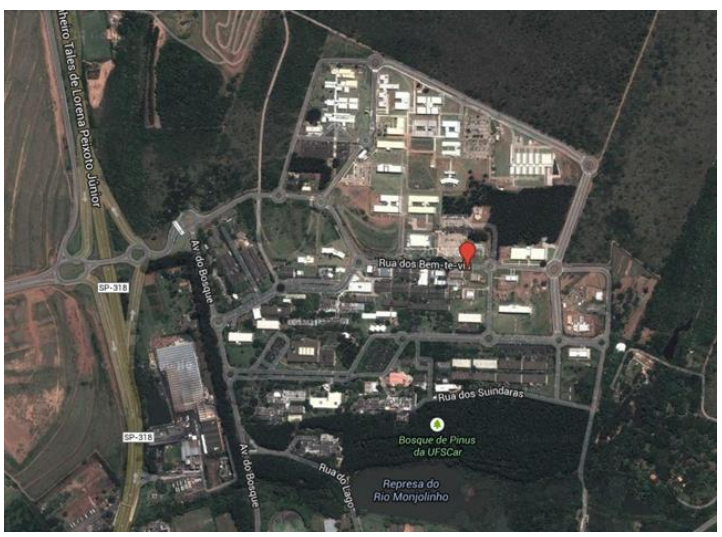

Fonte: Google Maps
Figura 2 - Planta baixa e corte
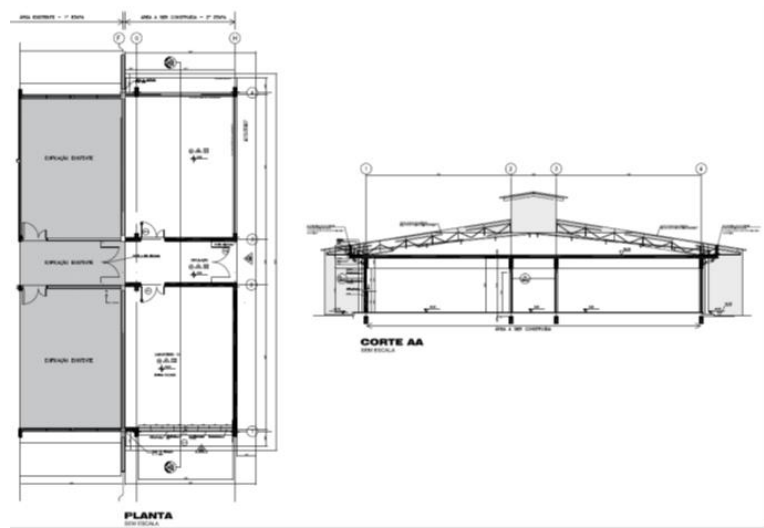

Foram utilizados neste estudo somente os projetos arquitetônico e estrutural que foram concebidos em softwares CAD 2D para fins tradicionais de licitação e construção da edificação. Para efeito de comparação, estes projetos da referida obra foram modelados em 3D para entendimento do processo de extração dos quantitativos a partir do BIM. Foram utilizados para a modelagem o software Sketchup de propriedade da empresa Google. Posteriormente, os dados obtidos via modelo 3D foram comparados com os quantitativos calculados manualmente que consideraram os critérios de medição estabelecidos no edital de licitação e no manual descritivo da obra elaborados pelos profissionais da Universidade.

Para a modelagem utilizou-se como base os projetos originais e foram seguidos os passos descritos na Figura 3. Durante a modelagem verificou-se que era importante que este passo a passo fosse estabelecido a partir da sequência tecnológica de construção de forma a favorecer a extração dos quantitativos para compor a planilha orçamentária da edificação.

Figura 3 - Passo a passo da modelagem em BIM

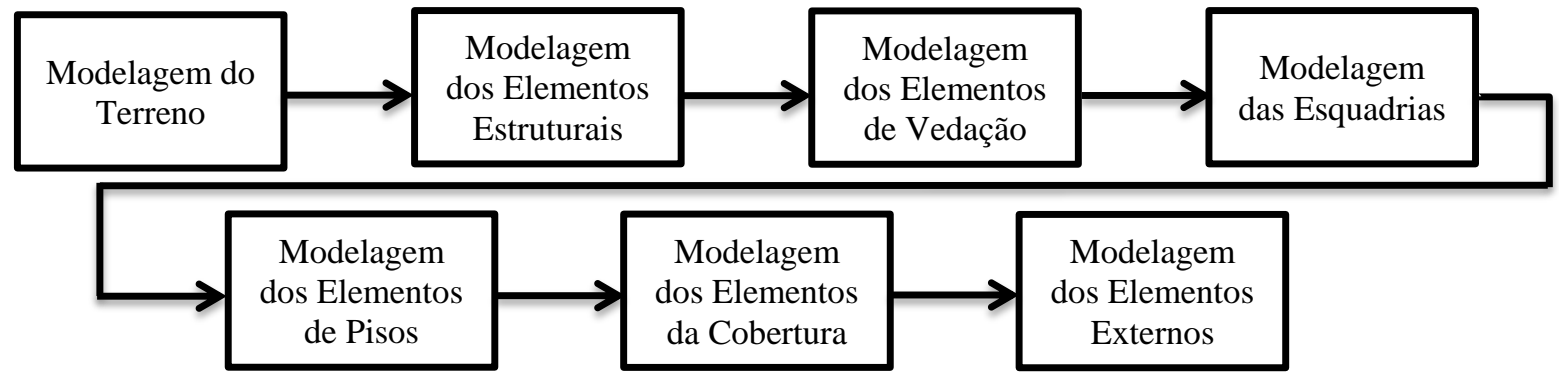

Fonte: Elaborado pelos autores

Para facilitar compreensão da comparação entre a forma tradicional com a forma BIM de se quantificar materiais e serviço, foi atribuída uma escala de ordem gradual que vai de Faltante $(\mathrm{F})$, Parcial $(\mathrm{P})$ e Equivalente $(\mathrm{E})$ para cada item presente na planilha orçamentária, seguindo o critério descrito na Quadro 2. 
Quadro 2 - Escala de ordem gradual para comparação dos orçamentos

\begin{tabular}{|c|c|l|}
\hline GRAU & LEGENDA & \multicolumn{1}{c|}{ DESCRIÇÃO } \\
\hline Faltante & F & $\begin{array}{l}\text { Quando a informação gerada pelo modelo não contribui para o } \\
\text { levantamento de quantitativos do item orçado. }\end{array}$ \\
\hline Parcial & P & $\begin{array}{l}\text { Quando a informação gerada pelo modelo é parcial para o } \\
\text { levantamento de quantitativos do item orçado. }\end{array}$ \\
\hline Equivalente & E & $\begin{array}{l}\text { Quando a informação gerada pelo modelo se equivale ao } \\
\text { levantamento de quantitativos do item orçado. }\end{array}$ \\
\hline
\end{tabular}

Fonte: Elaborada pelos autores

\section{RESULTADOS}

A Figura 4 ilustra os elementos da obra que foram modelados no 3D seguindo o passo a passo descrito anteriormente.

\section{Figura 4 - Ilustrações do Processo de Modelagem}

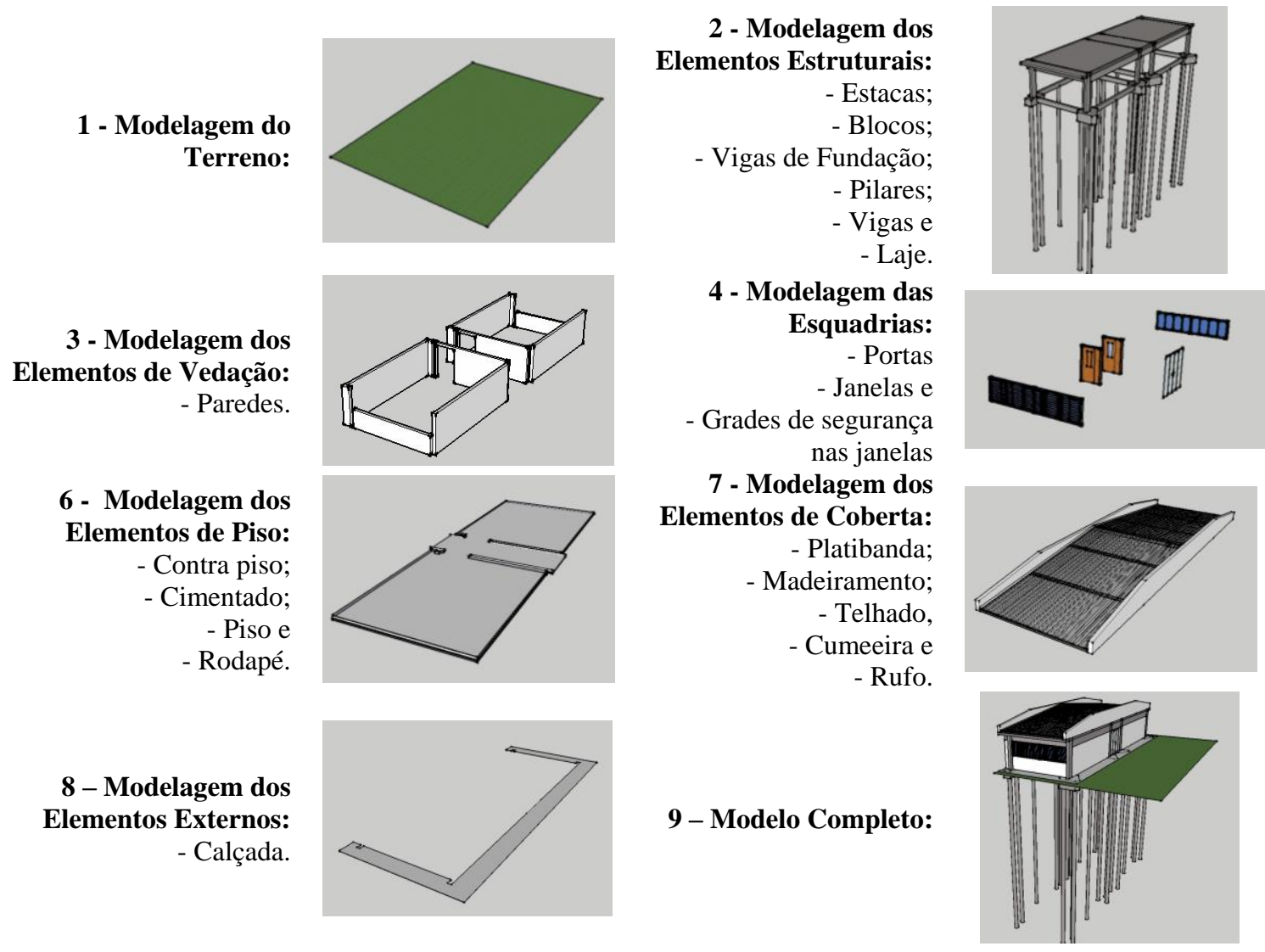

Fonte: Elaborada pelos autores

Para a comparação, além de obter o quantitativo a partir do modelo, foi feito uma análise de como foi obtido o valor quantitativo na metodologia tradicional, para conseguir um melhor entendimento e empregar as mesmas considerações no modelo, como descrito na Figura 5. 
Figura 5 - Parte da planilha de comparativo entre as metodologias

\begin{tabular}{|c|c|c|c|c|c|}
\hline \multicolumn{6}{|c|}{ CAMPARATIVO ENTRE AS METODOLOGIAS } \\
\hline \multirow{2}{*}{ ITENS } & \multirow{2}{*}{ DESCRIÇÃO } & \multirow{2}{*}{ UND } & \multirow{2}{*}{\begin{tabular}{|c|} 
TRADICIONAL \\
OBTENÇÃO DO DADO \\
\end{tabular}} & \multicolumn{2}{|r|}{ SKETCHUP } \\
\hline & & & & AVALIAÇÃO & OBTENÇÃO DO DADO \\
\hline 3.0 & INFRAESTRUTURA & & & & \\
\hline 3.1 & Estacas Strauss & & & & \\
\hline 3.1 .1 & $\begin{array}{l}\text { Estaca tipo strauss - moldada no local, } \\
\text { diâmetro de } 25,0 \mathrm{~cm} \text { - sem armação }\end{array}$ & $\mathrm{m}$ & \multirow{2}{*}{ Comprimento dos elementos } & \multirow{2}{*}{ E } & \multirow{2}{*}{$\begin{array}{l}\text { O modelo informa o } \\
\text { comprimento do } \\
\text { componente }\end{array}$} \\
\hline 3.1 .2 & $\begin{array}{l}\text { Estaca tipo strauss - moldada no local, } \\
\text { diâmetro de } 32,0 \mathrm{~cm} \text { - sem armação }\end{array}$ & $\mathrm{m}$ & & & \\
\hline 3.2 & Escavação Manual & & & & \\
\hline 3.2 .1 & $\begin{array}{l}\text { Escavação Manual em campo aberto de } \\
\text { solo (exceto rocha), com profundidade } \\
\text { de até } 2,00 \mathrm{~m}\end{array}$ & $\mathrm{~m}^{3}$ & $\begin{array}{l}\text { Multiplicação das dimensões dos } \\
\text { elementos acrescidas de uma } \\
\text { medida destinada a área de } \\
\text { montagem das formas }\end{array}$ & $\mathbf{P}$ & $\begin{array}{l}\text { Volume do elemento } \\
\text { acrescido do volume da área } \\
\text { necessária para fixação das } \\
\text { formas }\end{array}$ \\
\hline 3.3 & Regularização e apiloamento de fundo de & & & & \\
\hline 3.3 .1 & $\begin{array}{l}\text { Regularização e compactação manual de } \\
\text { terreno com soquete }\end{array}$ & $\mathrm{m}^{2}$ & Mesma área de escavação & $\mathbf{P}$ & $\begin{array}{l}\text { Área do elemento acrescida } \\
\text { da área necessária para } \\
\text { fixação das formas }\end{array}$ \\
\hline 3.4 & Lastro & & & & \\
\hline 3.4 .1 & Lastro de concreto & $\mathrm{m}^{3}$ & $\begin{array}{l}\text { Ára de fundo dos elementos } \\
\text { acrescidas de uma medida } \\
\text { destinada a área de montagem } \\
\text { das formas, multiplicada pela } \\
\text { espessura do lastro }\end{array}$ & $\mathbf{P}$ & $\begin{array}{l}\text { Área do elemento acrescida } \\
\text { da área necessária para } \\
\text { fixação das formas }\end{array}$ \\
\hline 3.5 & Forma para Fundações & & & & \\
\hline 3.5 .1 & $\begin{array}{l}\text { Forma de tabua para concreto em } \\
\text { fundação com } 2 \text { reaproveitamentos }\end{array}$ & $m^{2}$ & Área das faces dos elementos & E & $\begin{array}{l}\text { A área das face de forma é } \\
\text { retirada diretamente do } \\
\text { modelo }\end{array}$ \\
\hline 3.6 & Aço para Fundações & & & & \\
\hline 3.6 .1 & Fornecimento de aço CA-60, diâmetro de & $\mathrm{kg}$ & \multirow{5}{*}{$\begin{array}{l}\text { Comprimentos dos aços } \\
\text { multiplicado pela sua respectiva } \\
\text { massa a cada metro }\end{array}$} & \multirow{5}{*}{$\mathbf{F}$} & \multirow{5}{*}{$\begin{array}{l}\text { O software só da } \\
\text { informação de } \\
\text { comprimento, área e } \\
\text { volume, não da quilos. } \\
\text { Também não foi modelado } \\
\text { a armadura da estrutura }\end{array}$} \\
\hline 3.6 .2 & Fornecimento de aço CA-50, diâmetro de & $\mathrm{kg}$ & & & \\
\hline 3.6.3 & Fornecimento de aço CA-50, diâmetro de & $\mathrm{kg}$ & & & \\
\hline 3.6 .4 & Fornecimento de aço CA-50, diâmetro de & $\mathrm{kg}$ & & & \\
\hline 3.6 .5 & $\begin{array}{l}\text { Execução de corte, dobra, montagem e } \\
\text { colocação de ferragem na forma, aço CA- } \\
50 / 60 \text {, em barra redonda com diâmetro }\end{array}$ & $\mathrm{kg}$ & & & \\
\hline 3.7 & Concreto para Fundações & & & & \\
\hline 3.7 .1 & $\begin{array}{l}\text { Concreto usinado fck = } 25 \mathrm{mpa} \text {, inclusive } \\
\text { lançamento e adensamento }\end{array}$ & $\mathrm{m}^{3}$ & Volume dos elementos & E & $\begin{array}{l}\text { O modelo informa o volume } \\
\text { dos elementos }\end{array}$ \\
\hline 3.8 & Impermeabilização das fundações & & & & \\
\hline 3.8 .1 & $\begin{array}{l}\text { Chapisco traço 1:3 (cimento e areia } \\
\text { grossa), espessura 0,5 cm, preparo } \\
\text { mecânico da argamassa }\end{array}$ & $\mathrm{m}^{2}$ & $\begin{array}{l}\text { Área das faces } \\
\text { impermeabilizadas }\end{array}$ & E & $\begin{array}{l}\text { O modelo informa a área } \\
\text { das face que vai ser } \\
\text { impermeabilizada }\end{array}$ \\
\hline 3.8 .2 & $\begin{array}{l}\text { Impermeabilização de superfície com } \\
\text { argamassa de cimento e areia (média), } \\
\text { traço 1:3, com aditivo impermeabilizante, }\end{array}$ & $\mathrm{m}^{2}$ & $\begin{array}{l}\text { Área das faces } \\
\text { impermeabilizadas }\end{array}$ & E & $\begin{array}{l}\text { O modelo informa a área } \\
\text { das face que vai ser } \\
\text { impermeabilizada }\end{array}$ \\
\hline 3.8 .3 & $\begin{array}{l}\text { Impermeabilização de fundações, } \\
\text { baldrames, muros de arrimo, alicerces e } \\
\text { revestimento em contato com o solo - }\end{array}$ & $m^{2}$ & $\begin{array}{l}\text { Área das faces } \\
\text { impermeabilizadas }\end{array}$ & E & $\begin{array}{l}\text { O modelo informa a área } \\
\text { das face que vai ser } \\
\text { impermeabilizada }\end{array}$ \\
\hline 3.9 & Reaterro e Compactação & & & & \\
\hline 3.9 .1 & Reaterro manual de valas & $\mathrm{m}^{3}$ & $\begin{array}{l}\text { Volume de escavação menos o } \\
\text { volumedo de concreto e do } \\
\text { lastro de cada elemento }\end{array}$ & $\mathbf{P}$ & $\begin{array}{l}\text { Vai ser o volume escavado } \\
\text { menos a área do elemento } \\
\text { escavado }\end{array}$ \\
\hline
\end{tabular}

Fonte: Elaborada pelos autores

Para exemplificar, foram detalhados os serviço de escavação dos blocos e execução de piso granilite. A Tabela 1 apresenta o levantamento de quantitativos dos blocos, elaborado na forma tradicional. O serviço de escavação também consta desta tabela, sendo que o critério de medição adotado foi acrescentar $0,60 \mathrm{~m}$ aos lados de cada bloco como espaço para posicionamento das formas. O volume a ser escavado foi calculado como o somatório do volume dos blocos com o espaço para o posicionamento das formas, totalizando $21,02 \mathrm{~m}^{3}$. 
Tabela 1 - Quantitativo de escavação dos blocos (tradicional)

\begin{tabular}{|c|c|c|c|c|c|c|c|c|c|c|}
\hline \multirow{3}{*}{ DESCRIÇÃOO } & \multicolumn{10}{|c|}{ QUANTITATIVOS } \\
\cline { 2 - 11 } & Comp & Larg & Alt & Quant & $\begin{array}{c}\text { V. } \\
\text { Conc }\end{array}$ & $\begin{array}{c}\text { V. } \\
\text { Escav }\end{array}$ & Regula. & Lastro & $\begin{array}{c}\text { F. de } \\
\text { Táb. }\end{array}$ & Reaterro \\
\cline { 2 - 11 } & $(\mathbf{m})$ & $(\mathbf{m})$ & $(\mathbf{m})$ & un & $\left(\mathbf{m}^{3}\right)$ & $\left(\mathbf{m}^{3}\right)$ & $\left(\mathbf{m}^{2}\right)$ & $\left(\mathbf{m}^{3}\right)$ & $\left(\mathbf{m}^{2}\right)$ & $\mathbf{m}^{\mathbf{3}}$ \\
\hline B01 & 1,60 & 0,64 & 0,55 & 4,00 & 2,25 & 6,55 & 8,32 & 0,36 & 9,856 & 3,94 \\
\hline B02 & 1,41 & 1,80 & 0,70 & 4,00 & 4,72 & 14,47 & 15,93 & 0,72 & 14,45 & 9,03 \\
\hline & SUBTOTAL & & $\mathbf{6 , 9 8}$ & $\mathbf{2 1 , 0 2}$ & $\mathbf{2 4 , 2 5}$ & $\mathbf{1 , 0 8}$ & $\mathbf{2 4 , 3 0}$ & $\mathbf{1 2 , 9 7}$ \\
\hline
\end{tabular}

Fonte: Elaborada pelos autores

A Figura 6 mostra como foi feita a extração do volume dos blocos a partir do modelo $3 \mathrm{D}$, onde os mesmos foram destacados. Com isso, pode-se observar na caixa "Informações da entidade" do software que o volume dos blocos é de $6,986 \mathrm{~m}^{3}$. Entretanto, verifica-se que o quantitativo de escavação não é obtido diretamente, pois o volume dos espaços destinado ao posicionamento das formas não é representado na figura, dado que não pode ser gerado pelo modelo. Com isso, nesta análise, foi atribuído a este item o grau de "Parcial", já que o modelo gerou parte da informação necessária.

Figura 6 - Quantitativo do volume dos blocos (BIM)

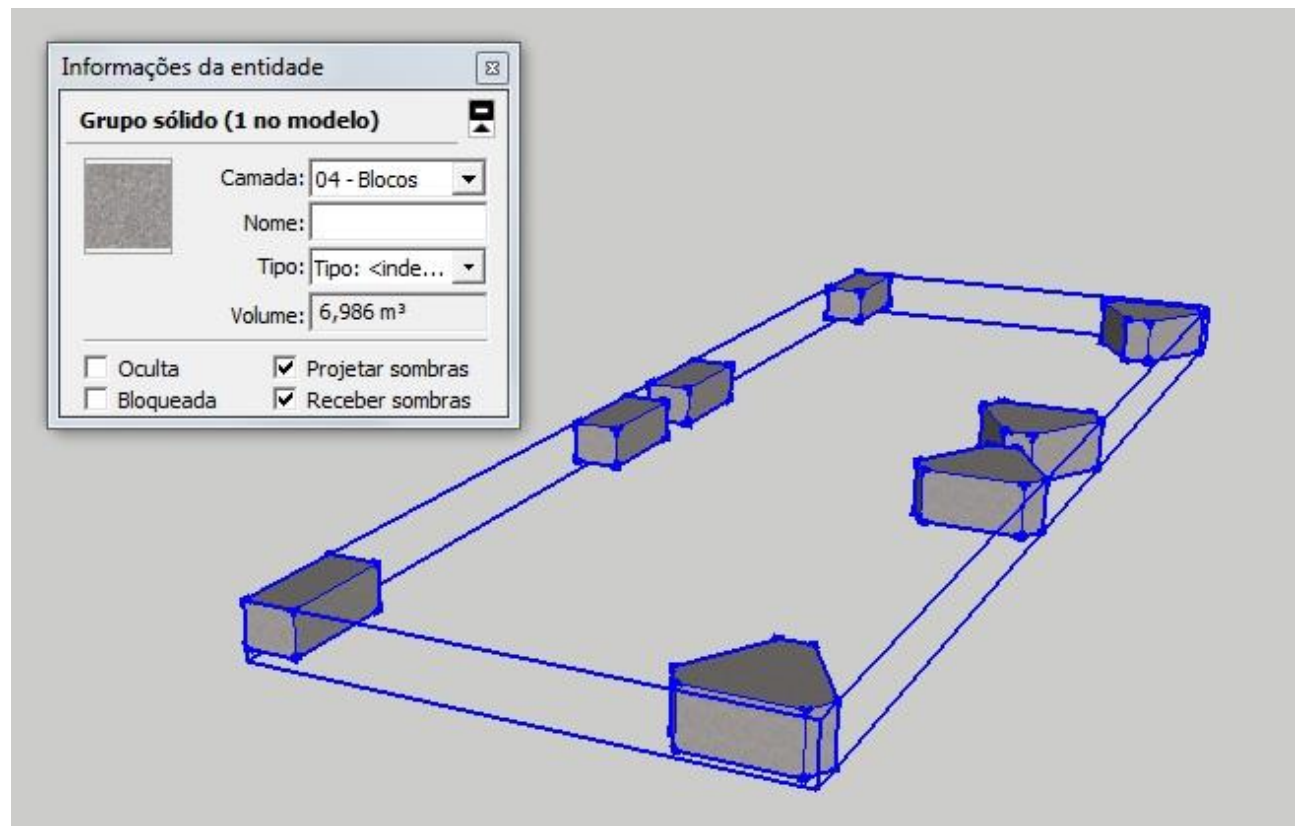

Fonte: Elaborada pelos autores

A planilha do levantamento tradicional de quantitativos da execução do piso granilite é mostrada na Tabela 2, onde se pode notar que foi encontrado uma área total de piso de $93,38 \mathrm{~m}^{2}$. Este total foi encontrado através do somatório das áreas de cada ambiente, estabelecida por meio da multiplicação do comprimento pela largura dos mesmos.

Tabela 2 - Quantitativo de execução de piso granilite (Tradicional)

\begin{tabular}{|c|c|c|c|c|c|}
\hline DESCRIÇÃO & \multicolumn{5}{|c|}{ QUANTITATIVOS } \\
\hline & Quant. & Comp. & Larg. & Área & Perimetro \\
\hline & und & m & m & m $^{\mathbf{2}}$ & m \\
\hline Pisos 01 e 03 & 2,00 & 7,40 & 5,44 & 80,51 & 50,16 \\
\hline Piso 02 & 1,00 & 2,31 & 5,57 & 12,87 & 11,18 \\
\hline \multicolumn{7}{|c|}{ TOTAL } & $\mathbf{9 3 , 3 8}$ & $\mathbf{6 1 , 3 4}$ \\
\hline
\end{tabular}

Fonte: Elaborada pelos autores 
A Figura 7 exibe a quantificação da execução do piso granilite gerada pelo modelo. Deste modo, observa-se que a área encontrada para execução de piso é de $93,098 \mathrm{~m}^{2}$, tendo uma diferença de $0,282 \mathrm{~m}^{2}$ para o procedimento tradicional, explicada pelo fato de não ter sido considerado os recortes dos pilares na quantificação tradicional. Para este item foi atribuído o grau de "Equivalente", já que o modelo gerou a informação necessária. De forma semelhante, a quantificação do granite no rodapé também foi considerada "Equivalente" nos dois processos.

\section{Figura 7 - Quantitativo de execução de piso granilite (BIM)}

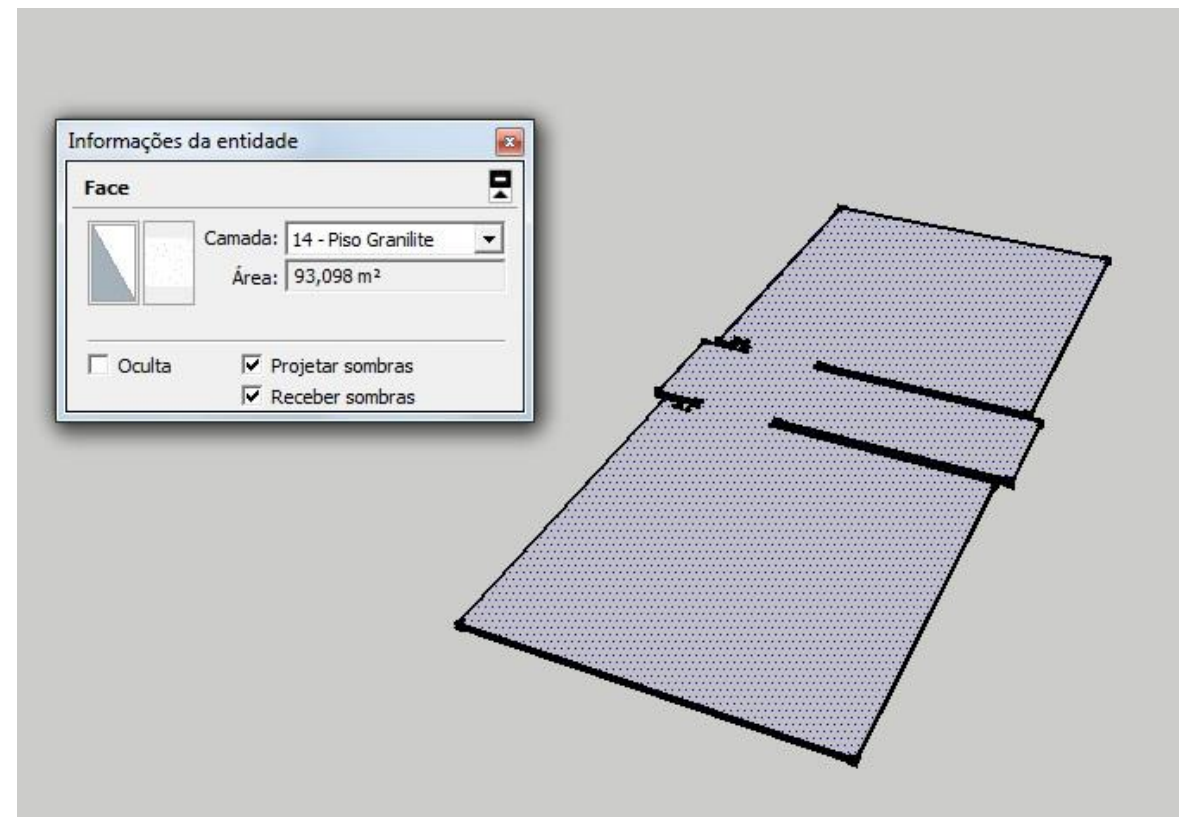

Fonte: Elaborada pelos autores

Estendendo a análise para todos os itens da planilha orçamentária foram encontrados os resultados de comparação apresentados na Tabela 3.

Tabela 3 - Resultado da escala de ordem gradual

\begin{tabular}{|l|c|c|}
\hline \multicolumn{1}{|c|}{ GRAU } & NÚMERO DE ITENS & PERCENTUAL \\
\hline Equivalente (E) & 39 & $63,93 \%$ \\
\hline Parcial (P) & 9 & $14,75 \%$ \\
\hline Faltante (F) & 13 & $21,31 \%$ \\
\hline Número total de itens & 61 & $100,00 \%$ \\
\hline
\end{tabular}

Fonte: Elaborada pelos autores

Deste modo, constatou-se que em $63,93 \%$ dos itens o modelo BIM forneceu a informação "Equivalente" (E) ao item da planilha orçamentária tradicional, demostrando a contribuição do modelo BIM para o processo de levantamento quantitativo, pois os mesmos são extraídos diretamente do modelo e tem um grau de precisão mais elevado.

Os itens "Parcial" (P) e "Faltante" (F) que tiveram 14,75\% e 21,31\% respectivamente, não podem ser considerados um grande percentual de itens não favoráveis ao BIM. 
Entende-se que os percentuais de incompatibilidades podem ser reduzidos se o grau de detalhamento do modelo for aumentado.

\section{CONCLUSÃO}

Os resultados indicaram que os quantitativos extraídos do BIM apresentam um significativo grau de confiabilidade e que a maioria dos itens da planilha orçamentária pode ser extraída de forma automática por meio do modelo BIM. Isto sugere que o BIM é uma ferramenta que não apenas facilita as atividades de projeto, mas também contribui para outras atividades, como para a etapa de levantamento de quantitativos do orçamento, eliminando alguns erros manuais comuns desta etapa e colaborando para uma melhor qualidade do orçamento.

\section{AGRADECIMENTOS}

À CAPES, pela bolsa de pesquisa, e à FINEP, projeto CANTECHIS, pelo apoio recebido.

\section{REFERÊNCIAS}

ANDRADE, L.S. A contribuição dos sistemas BIM para o planejamento orçamentário das obras públicas: estudo de caso do auditório e da biblioteca de Planaltina. 2012. 122 f. Dissertação (Mestrado em Arquitetura e Urbanismo) - Universidade de Brasília, Brasília, 2012. Disponível em: <http://repositorio.unb.br/bitstream/10482/10637/1/2012_LudmilaSantosAndrade.pdf>. Acesso em maio de 2014.

AZEVEDO, O.J.M. Metodologia BIM - Building Information Modeling na Direção Técnica de Obras. 82 p. Dissertação (Mestrado em Engenharia Civil) - Universidade do Minho. Braga. 2009.

BARBOSA, C. et al. Gerenciamento de custos em projetos. 3ª ed. Rio de Janeiro: Editora FGV, 2009.

EASTMAN, C.; TEICHOLZ, P.; SACKS, R.; LISTON, K. Manual de BIM: um guia de modelagem da informação da construção para arquitetos, engenheiros, gerentes, construtores e incorporadores. Porto Alegre: Bookman, 2014.

MATTOS, A. D. Como preparar orçamentos de obras: dicas para orçamentistas, estudo de caso, exemplos. São Paulo: Editora Pini, 2006.

SABOL, L. Challenges in cost estimating with Building Information Modeling. Design + Construction Strategic. 15p., 2008. Disponível em: <http://pdf-release.net/2/Challenges-in-Cost-Estimating-withBuilding-Information-Modeling>. Acesso em maio de 2014.

SANTOS, A.P.L.; WITICOVSKI, L. C.; GARCIA, L. E. M.; SCHEER, S. A utilização do BIM em projetos de construção civil. Iberoamerican Journal of Industrial Engineering (IJIE), Florianópolis, SC, vol.1, $\mathrm{n}^{\mathrm{o}} \quad 2, \quad$ p. 24-42, dez. 2009. Disponível em: <http://incubadora.periodicos.ufsc.br/index.php/IJIE/article/viewFile/171/pdf_49>. Acesso em maio de 2014.

WITICOVSKI, L.C. Levantamento de quantitativos em projeto: uma análise comparativa do fluxo de informações entre as representações em 2D e o modelo de informações da construção (BIM). 181 p. Dissertação (Mestre em Construção Civil) - Universidade Federal do Paraná, Curitiba, 2011. Disponibilizado em: m: http://dspace.c3sl.ufpr.br:8080/dspace/bitstream/handle/1884/27190/WITICOVSKI_DISSERTDISS_201 1.pdf? sequence $=1>$. Acesso em maio de 2014 . 